\title{
Characteristics of MODIS BRDF shape and its relationship with land cover classes in Australia
}

\author{
$\underline{\text { F. Li }}^{\text {a }}$, D. L. B. Jupp ${ }^{\text {b }}$ L. Lymburner ${ }^{\text {a }}$, P. Tan ${ }^{\text {a }, ~ A . ~ M c I n t y r e ~}{ }^{\text {a }}$, M. Thankappan ${ }^{\text {a }}$, \\ A. Lewis ${ }^{a}$ and A. Held ${ }^{b}$ \\ ${ }^{a}$ National Earth Observation Group, Geoscience Australia, GPO Box 378, ACT, 2601, Australia \\ ${ }^{b}$ CSIRO Marine and Atmospheric Research, GPO Box 3023, ACT 2601, Australia
}

Email: Fuqin.Li@ga.gov.au

\begin{abstract}
Surface Bidirectional Reflectance Distribution Function (BRDF) correction of spectral data (Li et al., 2010) has important applications to time series based analysis and classification. However, it has been reasonably proposed that the BRDF information itself can be used directly in the time series applications for land cover mapping and climate change etc. To use such data it is important to understand the characteristics of BRDF and its variation over different cover and climate conditions and how they relate to well-understood variations in spectral data in terms of the land cover characteristics and changes. Many studies have suggested that BRDF is related to the characteristics of land cover types (Brown de Colstoun and Walthall, 2006 and Jiao et al., 2011), especially to vegetation structure (height and cover) (Lovell and Graetz, 2002; Li et al., 2013) and also climate patterns. In this study, 10 years of MODIS BRDF data sets (MOD43A1) from 2002 to 2011 have been used to conduct an analysis using time series data for land cover data products available in Australia. The data have been averaged over individual years to remove the seasonal patterns and variation for reasons which were outlined in Li et al. (2013) and are briefly discussed later in this paper. Using the "root mean square" (RMS, the distance of the shape function from Lambertian which is a measure of its asymmetry) as a BRDF shape indicator, with the inter-annual data series the study has found that:
\end{abstract}

- The average $R M S$ for three bands (red, near-infrared and shortwave infrared) for each year is well correlated with Normalized Difference Vegetation Index (NDVI) if it is separated by land cover classes. Correlation coefficients $\mathrm{R}^{2}$ range between $0.5-0.7$. The $R M S$ also varies significantly between land cover classes.

- Inter-annual variation of $R M S$ is small for typical vegetation classes, especially for classes with high vegetation cover.

- If Normalized Difference $R M S$ is used (called NDRMS, calculated using red and near-infrared bands and the same formula as $N D V I$ ), its relationship with $N D V I$ is much stronger than that of $R M S$. Correlation coefficients $\mathrm{R}^{2}$ are close to 0.9 for most of the years. Each land cover class has well defined NDRMS patterns. The separation is clearer than for the $R M S$ patterns.

- NDRMS seems quite sensitive to climate change as indicated by NDVI but the relationship over the 10 years in some classes is different from the overall relationship between classes averaged over all years. In vegetated classes, NDRMS has tended to increase in this way much more sensitively after the change from a long dry period to wet years, and most particularly after 2009. The sensitivity has apparently increased with class average $N D V I$.

From the above, it has been concluded that:

- Both RMS and NDRMS are able to differentiate land cover classes defined in the Australian Dynamic Land Cover Dataset (DLCD) series well. They both correlate well with spectral NDVI if the patterns are separated by land cover classes and averaged at least over individual years (removing intra-annual effects).

- Both RMS and NDRMS can potentially be used as additional features to map land cover. However, $N D R M S$ seems to be the more sensitive of the two.

- However, confident and successful use of these features will need additional understanding of the sources of the variation and the information they bring compared with traditional spectral data. In particular, further studies are needed to understand the rising sensitivity in NDRMS compared with NDVI as cover and greenness increase and the previously reported (Li at al., 2013) questions concerning relative phases of intra-annual variation in NDRMS and $R M S$ relative to $N D V I$.

Keywords: MODIS, BRDF shape function, land cover 


\section{INTRODUCTION}

The work reported here had its base in the practical choice for BRDF parameters to correct pre-MODIS Landsat data over the Australian continent. Studies of the MODIS BRDF parameters between 2001 and 2011 (Li et al., 2013) showed that the inter-annual variation was small compared with intra-annual (seasonal) variation. Therefore, the default models were derived by averaging 46 "weekly" sets of parameters over the 11 years. The variation between various sets of land cover classes was also much higher than the inter-annual variation indicating that spatial variation in the model was highly significant leading to the choice of leaving the definitions at the base $500 \mathrm{~m}$ spatial resolution. However, while the BRDF shape and its patterns are important parameters for correcting the BRDF effect on satellite data (Li et al., 2010, 2012), they also provide potential independent features to use for land cover mapping. As additional information, Sandmeier and Deering (1999), Brown de Colstoun and Walthall (2006), Hill, et al. (2008) and Jiao et al. (2011) have found that BRDF shape features can improve classification accuracy for some sparse or open land cover classes that are dominated by surface scattering. However, the wider utilisation of this opportunity has been limited.

To use such data it is important to understand the characteristics of BRDF and its variation over different cover and climate conditions and how they relate to well-understood variations in spectral data in terms of the land cover characteristics and changes. Many studies have found that there is a strong relationship between BRDF shape, vegetation structure and land cover types. For example, Lovell and Graetz (2002) found that there is a strong relationship between vegetation structure and BRDF parameters in Australia based on POLarization and Directionality of the Earth's Reflectance (POLDER) data. Li et al. (2013) also found that strong correlation exists between BRDF shape indicators (RMS/NDRMS) and vegetation structure using MODIS BRDF data.

Following on from the work reported by Li et al. (2013), in this study, further analysis has been conducted using a 10-year (2002-2011) MODIS BRDF time series and a corresponding series of land cover maps developed in Australia. The objective is to establish relationships between the spectrally derived classes in terms of BRDF and to investigate whether MODIS BRDF parameters can be used as additional information to improve land cover classification accuracy in Australia.

\section{DATA AND METHODS}

\subsection{Method}

Models for the surface bi-directional reflectance factor, $\rho_{s}\left(\theta_{S}, \theta_{V}, \varphi\right)$ can be simplified and expressed using kernel functions as summarised in a convenient context by Schaaf et al. (2002) as:

$\rho_{S}\left(\theta_{S}, \theta_{V}, \varphi\right)=F_{\text {iso }}\left[1+\frac{F_{v o l}}{F_{\text {iso }}} K_{v o l}+\frac{F_{\text {geo }}}{F_{\text {iso }}} K_{g e o}\right]=F_{\text {iso }} B\left(\theta_{S}, \theta_{V}, \varphi, \alpha_{1}, \alpha_{2}\right)$

In this expression, $\theta_{S}$ is solar zenith angle and $\theta_{V}$ is view zenith angle, $\varphi$ is relative azimuth between the sun and view directions. $F_{i s o}$ is the weight for the isotropic contribution; $F_{v o l}$ and $F_{g e o}$ are the weights for volume scattering and geometrical optical scattering contributions; and $K_{v o l}$ and $K_{\text {geo }}$ are volume scattering and geometrical optical scattering kernel functions. $K_{v o l}$ and $K_{\text {geo }}$ are functions of $\theta_{S}, \theta_{V}$ and $\varphi . B\left(\theta_{S}, \theta_{V}, \varphi, \alpha_{1}, \alpha_{2}\right)$ is the BRDF shape function where $\alpha_{1}$ is defined as $F_{\text {vol }} / F_{\text {iso }}$ and $\alpha_{2}$ is defined as $F_{\text {geo }} / F_{\text {iso }}$. The MODIS BRDF product is a time series of estimates for the coefficients $\left(F_{i s o}, F_{v o l}\right.$ and $\left.F_{g e o}\right)$.

Several BRDF shape indicators have been developed and used for analysis (Jiao et al., 2011). We are assuming (based on simple models) that NDVI is sensitive to leaf area index (LAI), but not structure, and BRDF shape is sensitive to both LAI and structure. The shape of the red and near-infrared BRDF is observed to change differently with LAI and structure so there is a spectral effect in BRDF shape. Based on these assumption, in this study, a statistic called the root mean square $(R M S)$ is used as the BRDF shape indicator to analyse anisotropy following the work done by Li et al. (2013). $R M S$ between two shape functions $\left(S_{l}\right.$ and $\left.S_{2}\right)$ is calculated as:

$$
R M S=\left(\frac{4}{\pi} \int_{0}^{\pi} \int_{0}^{\pi / 2} \int_{0}^{\pi / 2} \cos \theta_{S} \sin \theta_{S} \cos \theta_{V} \sin \theta_{V}\left|S_{1}-S_{2}\right|^{2} d \theta_{V} d \theta_{S} d \varphi\right)^{1 / 2}
$$

In this paper we have used the single shape statistic defined by taking $S_{I}$ as the target BRDF shape, $B$, as expressed in Eq. (1) and the second $\left(S_{2}\right)$ as the Lambertian shape function (equal to 1.0). RMS then becomes the distance of the shape function from Lambertian which is a measure of its asymmetry. 
To enhance the effect, an index (called NDRMS) which combines the red and near-infrared $R M S$ was also used. The calculation of NDRMS is similar to the NDVI, but uses RMS instead of surface reflectance and is expressed as:

$$
N D R M S=\frac{R M S_{r e d}-R M S_{\text {nir }}}{R M S_{r e d}+R M S_{\text {nir }}}
$$

Where $R M S_{\text {red }}$ and $R M S_{\text {nir }}$ are the $R M S$ statistics for the red and near-infrared bands respectively.

\subsection{MODIS BRDF data and pre-processing}

The MODIS BRDF group provides 500m MODIS BRDF (MCD43A) model parameters $\left(F_{i s o}, F_{v o l}\right.$ and $\left.F_{\text {geo }}\right)$ at overlapping 8-day "weekly" intervals based on 7 spectral bands. The data selected for this study were from 2002 to 2011 (10 years) for the Australian region and each year has 46 time periods. The product (Schaaf et al., 2002) is the result of fitting a model to samples from 16 days of atmospherically corrected MODIS surface reflectance data but sampling at 8 days. A combination of the Ross Thick volume kernel and the Lisparse Reciprocal (Schaaf et al., 2002) geometric kernel models is used to approximate these data.

However, there is considerable extraneous spatial and temporal variation in these data at their base resolution. It seems to be due to a range of factors, including mis-registration, limited available samples in terms of number and distribution, shade and occlusion effects in mountainous areas, sub-pixel cloud, BRDF variance and other residual effects. These seem to be amplified by instability in the model fitting which apparently can occur even when the quality flag is satisfactory. As a result of these factors, many outliers occur in the data that need to be removed before further analysis can be conducted. Many, but not all of the effects can be reduced by using only the best quality data as indicated in the MODIS quality flag metadata. These remain outliers are still need to be removed by statistical filtering.

In this study, three MODIS bands are used to calculate RMS. They are

Table 1. MODIS bands used in this study

\begin{tabular}{|c|c|}
\hline $\begin{array}{c}\text { MODIS } \\
\text { band }\end{array}$ & $\begin{array}{c}\text { Bandwidth } \\
\text { (nanometers) }\end{array}$ \\
\hline Band 1 & $620-670$ \\
\hline Band 2 & $841-876$ \\
\hline Band 7 & $2105-2155$ \\
\hline
\end{tabular}
MODIS band 1 (red), band 2 (near-infrared) and band 6 (shortwave infrared). To ensure greatest stability of the MODIS BRDF data, only the data using information from both Aqua and Terra have been used (i.e. data processed since 2002). Details of the MODIS bands used in this study and their spectral information are listed in Table 1.

\subsection{Land cover maps}

The time series of land cover maps for 2002-2011 were provided by Geoscience Australia. The first version of the base land cover map (The National Dynamic Land Cover Dataset, DLCD) was released in 2011 (Lymburner, et al., 2011). The products classify Australian land cover into 33 categories, which conform to 2007 International Standards Organization (ISO) Land Cover Standard (19144-2). They have been based on MODIS 250m Enhanced Vegetation Index (EVI) time series data. The land cover maps used in this study are the second version of the maps. It is an annual land cover series and is currently being evaluated. They comprise 10 yearly time series based land cover maps. Each map is produced using data from the preceding two-year period with a one-year overlap between maps. Three steps were used to generate the land cover maps. In the first step, noisy and invalid data points are removed from the time series. Secondly, a feature extraction algorithm converts time series into a set of 12 time series coefficients related to ground phenomena such as average greenness and plant phenology. In the last step, clustering processes based on a tailored support vector clustering algorithm are applied to subsets of the coefficients. The resultant clusters then form the basis for a further modeling process incorporating auxiliary data to generate the final DLCD. For further details, see Lymburner et al. (2011) and Tan et al. (2013).

\section{RESULTS AND DISCUSSION}

The $F_{\text {iso }}$ parameters were extracted and the $R M S$ statistics calculated for three bands using Eq. (2). The results were then averaged for each year. Figure 1 plots $R M S$ (average red, near-infrared and shortwave infrared bands) and NDRMS inter-annual variation for eight typical land cover classes from 2002 to 2011. 
Inter-annual variation of $R M S$ (Figure 1a) is relatively small, especially for vegetation classes with high cover. The sparse and scattered vegetation classes show more fluctuation. For these classes, background (e.g., grass, soil) change due to the seasonal climate variation (mainly annual rainfall in Australia) for each different year contributes to the $R M S$ variation (Roderick et al., 2004). The NDRMS provides a different story. In general NDRMS also has little inter-annual variation in the first few years, but after 2007 and particularly after 2009 it suddenly has an increasing trend (Figure 1b). The trend is not observed by RMS or NDVI. Since the climate patterns shifted after a long dry to wet in Australia over the same period, it would seem possible that NDRMS is more sensitive to climate change effects in land cover. There have certainly been significant changes in cover of grasses and some shrub growth in many parts of Australia in that period. However, at this time it is not yet clear if these data are displaying it. The inter-annual variation patterns of $R M S$ and $N D R M S$ for the DLCD land cover classes are similar to those of some older structural vegetation structure classes. Li et al. (2013) found that there was strong correlation between NDVI and RMS/NDRMS if the RMS and NDRMS patterns were separated by these vegetation structure classes. Consequently, the same analysis has been conducted in this study using the DLCD land cover classes. Additional analysis was also conducted for individual years to see whether the relationship also has inter-annual variation.

Figure 2 provides xy plots for $N D V I$ and $R M S$ for the period 2002-2011 for 33 land cover classes in Australia. The Figure shows that the relationship between $R M S$ and $N D V I$ is stable although there is clearly independent information in the two variables on a base of general correlation. The patterns are similar in the two plots and there is relatively small interannual variation during the 10-year period.

For further analysis, the correlation coefficient has been calculated for each year and is listed in Table 2. Table 2 shows that there is strong correlation between $R M S$ and NDVI. Although the $\mathrm{R}^{2}$
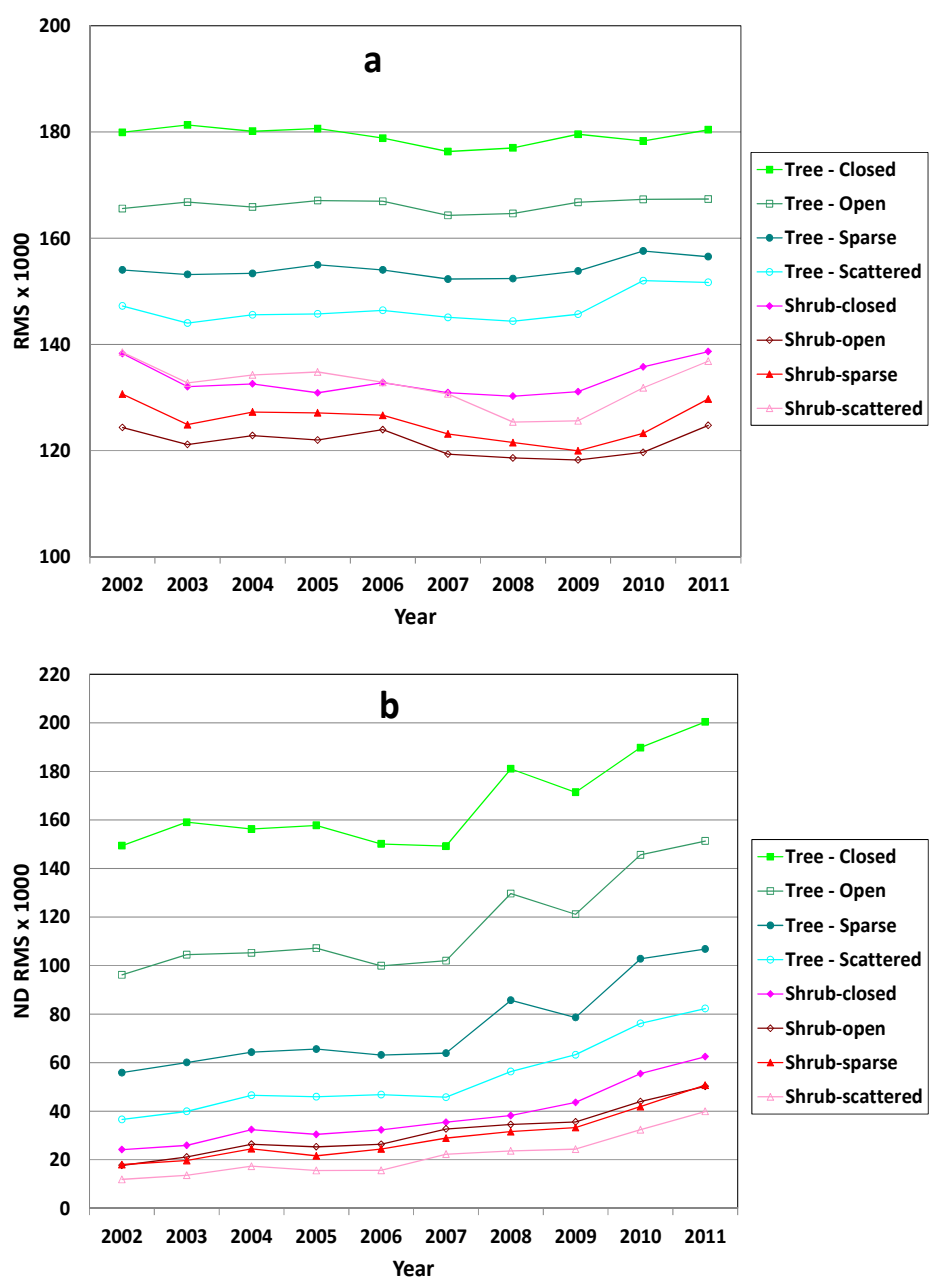

Figure 1. Inter-annual variation for 8 typical land cover classes in Australia for (a) RMS and (b) NDRMS
Table 2. Correlation coefficients between NDVI and RMS/NDRMS for 10 individual years.

Where: $R M S / N D R M S=\mathrm{a}+\mathrm{b} N D V I . \mathrm{R}^{2}$ is correlation coefficient.

\begin{tabular}{|c|c|c|c|c|c|c|}
\hline \multirow{2}{*}{ Year } & \multicolumn{3}{|c|}{$R M S$} & \multicolumn{3}{c|}{ NDRMS } \\
\cline { 2 - 7 } & $\mathrm{a}$ & $\mathrm{b}$ & $\mathrm{R}^{2}$ & $\mathrm{a}$ & $\mathrm{b}$ & $\mathrm{R}^{2}$ \\
\hline 2002 & 121.1 & 0.0839 & 0.5065 & -27.223 & 0.2604 & 0.923 \\
\hline 2003 & 115.63 & 0.0882 & 0.4945 & -27.717 & 0.2504 & 0.8272 \\
\hline 2004 & 112.95 & 0.0931 & 0.5949 & -24.285 & 0.2473 & 0.8681 \\
\hline 2005 & 115.98 & 0.0847 & 0.5915 & -25.514 & 0.2594 & 0.8789 \\
\hline 2006 & 110.47 & 0.1013 & 0.6313 & -26.066 & 0.2532 & 0.8433 \\
\hline 2007 & 111.42 & 0.0940 & 0.5865 & -17.636 & 0.2387 & 0.8521 \\
\hline 2008 & 109.21 & 0.0993 & 0.6817 & -14.166 & 0.2592 & 0.9032 \\
\hline 2009 & 105.35 & 0.1148 & 0.7109 & -14.381 & 0.2634 & 0.9158 \\
\hline 2010 & 111.15 & 0.0965 & 0.6424 & -13.759 & 0.2927 & 0.878 \\
\hline 2011 & 112.98 & 0.1023 & 0.6493 & -14.095 & 0.3041 & 0.8796 \\
\hline $2002-2011$ & 112.42 & 0.0964 & 0.6252 & -20.132 & 0.2621 & 0.8868 \\
\hline
\end{tabular}


changed each year (ranging between 0.5 and 0.7), the intercept (a) and slope (b) coefficients do not vary a great deal for the 10 -year period. It seems that $R M S$ is stable in regard to inter-annual variation.

Since Figure 1 suggests that NDRMS is quite sensitive to climate change and has stronger inter-annual variation compared with NDVI and RMS, the same analysis was also conducted for NDRMS. The right half of Table 2 shows the statistical results for NDVI vs NDRMS. The results show that the relationships between NDVI and NDRMS are much stronger than those of NDVI and RMS. The correlation coefficients $\mathrm{R}^{2}$ range between 0.85 and 0.92 . Both intercept (a) and slope (b) coefficients vary significantly between the first 5 years and the last five years, particularly in 2010 and 2011.

Figure 3 provides the xy plot for NDVI and NDRMS. There are some negative $N D R M S$ values, all of which are from inland water bodies where NDRMS is either negative or a very small number. It is possible that $N D R M S$ could be used as an additional parameter to improve water body mapping. Compared with Figures $3 \mathrm{a}$ and $3 \mathrm{~b}$, and as indicated numerically in Table 2, NDRMS increases significantly after 2007 and both the slope and the intercept of the relationship with $N D V I$ between classes has increased over the last 5 years.
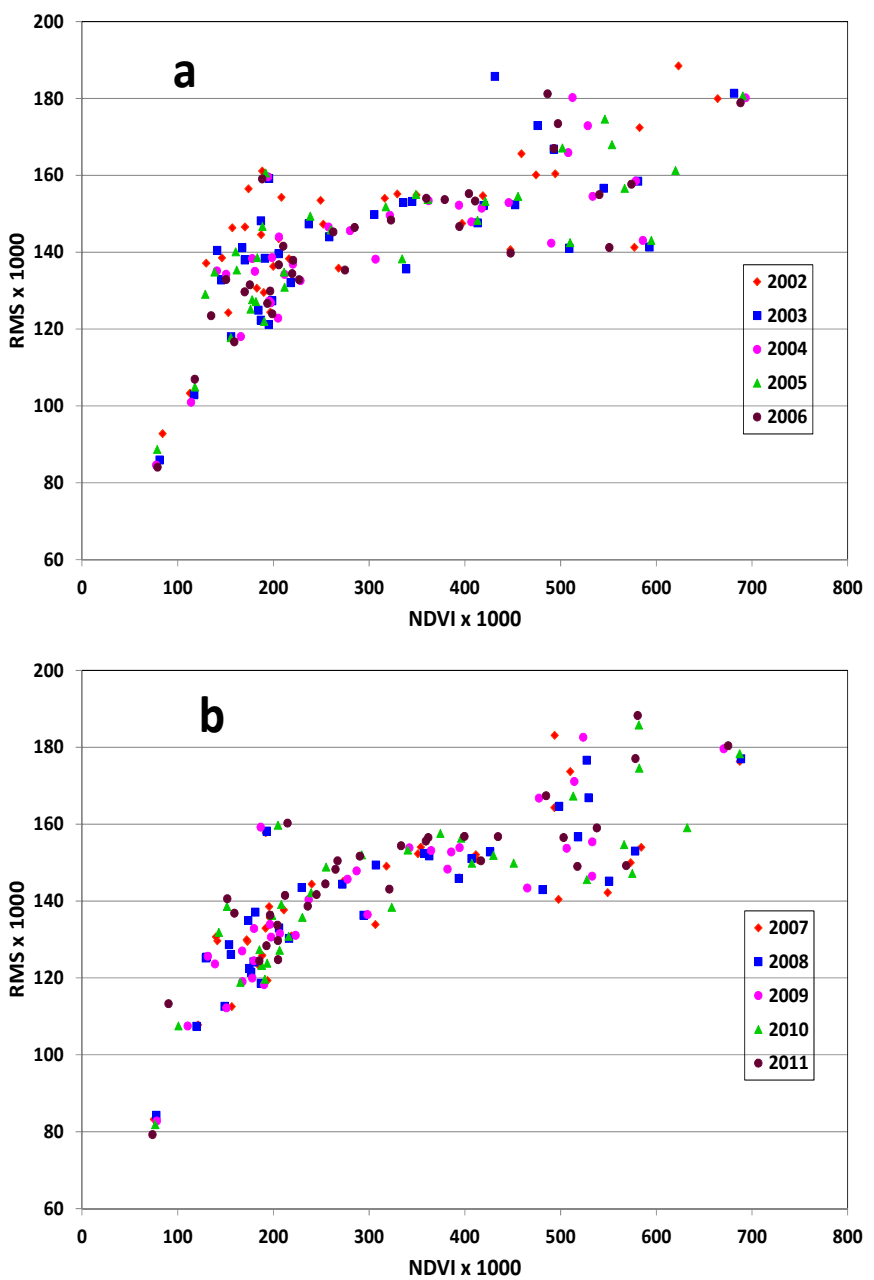

Figure 2. The relationship between NDVI and RMS for 20022011, separated into early and late as (a) 2002-2006 and (b)
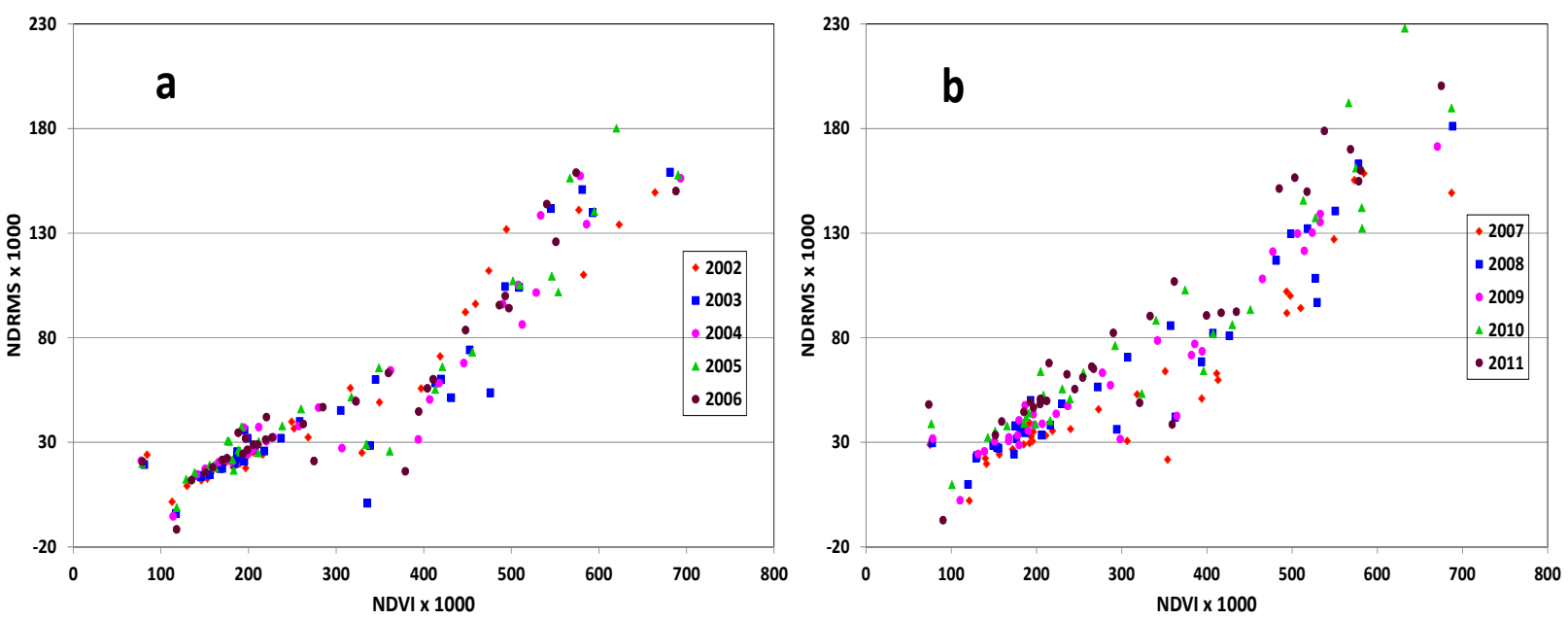

Figure 3. The relationship between NDVI and NDRMS for 2002-2011, separated into early and late as

(a) 2002-2006 and (b) 2007-2011

Comparing Figure 3 with Figure 2, it seems that there is strong clustering in Figure 3 when NDVI values are between 0.15 to 0.25 , but this does not appear in Figure 2, nor in the other NDVI range in Figure 3. Areas of low NDVI are mainly stable areas with little vegetation such as interior and semi-arid areas. But higher NDVI areas are likely to change much more - especially in green grass background. NDRMS is also expected to 
change with structure. That is the potential difference of the NDRMS relationship of Table 2. But so far, we do not have direct validation and it remains to be done.

If $R M S$ and NDRMS are averaged over the full 10 years, the overall relationship between $R M S / N D R M S$ is obtained as shown in Figure 4 and last row of Table 2. The Figure and the table show that the overall relationship is persistent, with the $\mathrm{R}^{2}$ being 0.6252 and 0.8868 for $R M S$ and NDRMS, respectively. The constants (a) and (b) are similar to those of the individual years for $R M S$, but for NDRMS, (a) and (b) are the average of first 5 and the later five years. They are obviously different from the last five years. The plot also shows a non-linear increase in sensitivity over NDVI as the cover of the

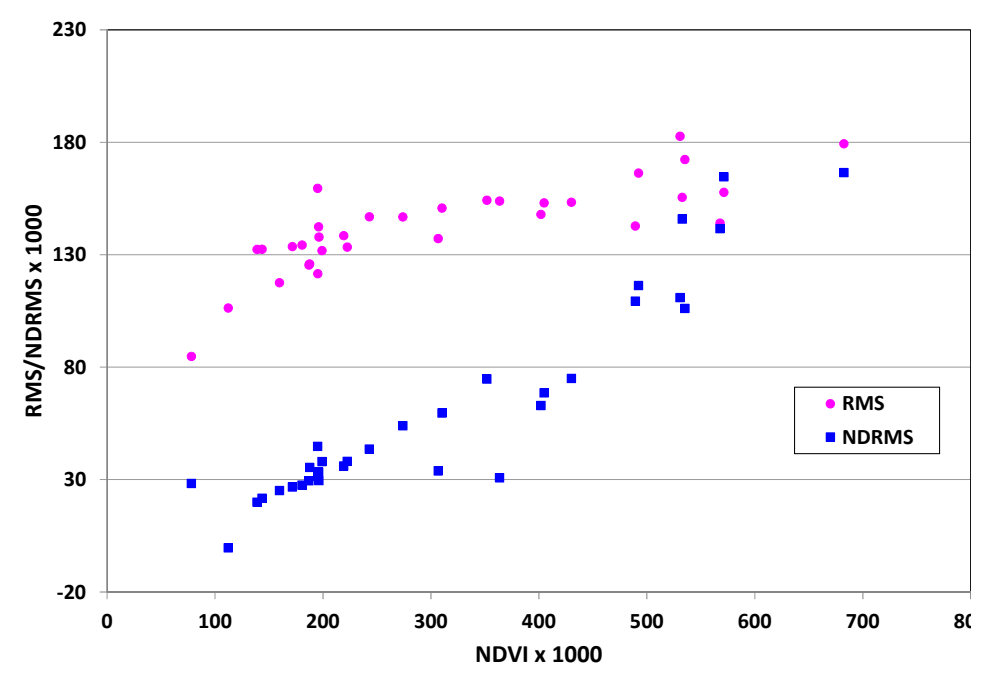

Figure 4. XY plot for 10 years average of $N D V I$ and $R M S / N D R M S$ class increases. This is consistent with the results seen in Table 2.

\section{CONCLUSIONS}

From the above analysis, we have found that BRDF spectral shape anisotropy (as expressed by $R M S$ and $N D R M S$ ) is well correlated with $N D V I$ at a yearly (inter-annual) scale, and BRDF shape is significantly different between land cover classes suggesting it provides useful information for discriminating land covers. The correlation between NDVI and NDRMS is stronger than that between NDVI and RMS. NDRMS has also significantly increased after 2009 but it is not yet confirmed that the trend is related functionally to the land cover response to inter-annual climate change. NDRMS also seems to be quite sensitive to water bodies and could possibly be used to distinguish their presence on the land surface.

NDRMS as a function of NDVI apparently changes in sensitivity to changing cover over time in vegetation classes. However, this still needs to be independently related to vegetation growth and changes in structure or dominant vegetation types. This must occur if it is to be used with confidence for land cover mapping. In addition, different behaviours of the statistics at the seasonal or intra-annual scale (as reported by Li et al., 2013) are being investigated. Intra-annual spectral data (e.g. NDVI and Albedo) display seasonal cycles that are well understood in terms of climate forcing of plant growth, evaporation and wetting/drying phases. But at the present time, while the RMS and NDRMS data also show seasonal relationships they have phase shifts that also need independent identification to be used confidently in seasonal monitoring.

Our conclusions at this stage of the investigation are:

I. Both RMS and NDRMS separate different land cover classes of the Australian DLCD series well. They also both correlate well with spectral NDVI if the patterns are separated by land cover classes and averaged at least over individual years (removing intra-annual effects).

II. Both RMS and NDRMS can potentially be used as additional features to map land cover. However, $N D R M S$ seems to be the more sensitive of the two.

III. However, confident and successful use of these features will need additional understanding of the sources of the variation and the information they bring compared with traditional spectral data. In particular, further studies are needed to understand the rising sensitivity in NDRMS compared with $N D V I$ as cover and greenness increase in some vegetated classes, and the previously reported ( $\mathrm{Li}$ at al., 2013) questions concerning relative phases of intra-annual variation in NDRMS and $R M S$ relative to $N D V I$.

\section{ACKNOWLEDGMENTS}

This paper is published with the permission of the CEO, Geoscience Australia (GA) and the permission of CSIRO. Access to MODIS BRDF data has been facilitated by Edward King and Matt Paget at CSIRO Marine \& Atmospheric Research (CMAR) who provided the MODIS BRDF time series data for Australia on 
an appropriate map base. Geoscience Australia provided series land cover maps. Ms Jenny Lovell of CSIRO, Mr Joshua Sixsmith and Mr Steven Curnow from GA reviewed the early draft of paper. Anonymous reviewers and editor provided many constructive comments and valuable suggestions that significantly improved the paper.

\section{REFERENCES}

Brown de Colstoun, E.C and Walthall, C.L (2006). Improving global scale land cover classifications with multi-directional POLDER data and a decision tree classifier. Remote Sensing of Environment, 100: 474485

Hill, M.J., Averill, C., Jiao, Z., Schaaf, C.B. and Armston, J.D. (2008). Relationship of MISR RPV parameters and MODIS BRDF shape indicators to surface vegetation patterns in an Australian tropical savannah. Canadian Journal of Remote Sensing, 34, S247-S267.

Jiao, Z., Woodcock, C., Schaaf, C.B., Tan, B., Liu, J., Gao, F., Strahler, A., Li, X. and Wang, J. (2011). Improving MODIS land cover classification by combining MODIS spectral and angular signatures in a Canadian boreal forest, Canadian Journal of Remote Sensing, 37 (2), 1-20.

Li, F., Jupp, D.L.B., Reddy, S., Lymburner, L., Mueller, N., Tan, P. and Islam, A. (2010). An evaluation of the use of atmospheric and BRDF correction to standardize Landsat data. The IEEE Journal of Selected Topics in Applied Earth Observations and Remote Sensing, 3, 257-270

Li, F., Jupp, D.L.B., Thankappan, M., Lymburner, L., Mueller, N., Lewis, A. and Held, A. (2012). A physicsbased atmospheric and BRDF correction for Landsat data over mountainous terrain. Remote Sensing of Environment, 124, 756-770

Li, F., Jupp, D.L.B., Thankappan, M., Paget, M, Lewis, A. and Held, A. (2013). The variability of satellite derived surface BRDF shape over Australia from 2001-2011. IEEE International Geoscience and Remote Sensing Symposium, Melbourne, Australia.

Lovell, J. L. and Graetz, R.D. (2002). Analysis of POLDER-ADEOS data for the Australian continent: The relationship between BRDF and vegetation structure. International Journal of Remote Sensing, 23, 27672796.

Lymburner, L., Tan, P., Mueller, N., Thackway, R., Lewis, A., Thankappan, M., Randall, L., Islam, A. and Senarath, U. (2011). The National Dynamic Land Cover Dataset - Technical report, Geoscience Australia, https://www.ga.gov.au/products/servlet/controller?event=GEOCAT_DETAILS\&catno=71069

Roderick, M.L., Noble, I.R. and Cridland, S.W. (1999). Estimating woody and herbaceous vegetation cover from time series satellite observations. Global Ecology and Biogeography, 8, 501-508.

Sandmeier, S. and Deering, D.W. (1999). Structure analysis and classification of boreal forests using hyperspectral BRDF data from ASAS. Remote Sensing of Environment, 69, 281-295

Schaaf, C., Gao, F., Strahler, A.H., Lucht, W., Li, X., Tsang, T., Strugnell, N.C., Zhang, X., Jin, Y., Muller, J.P., Lewis, P., Barnsley, M., Hobson, P., Disney, M., Roberts, G., Dunderdale, M., Doll, C., d'Entremont, R.P., Hu, B., Liang, S., Privette, J.L. and Roy, D. (2002). First operational BRDF, albedo and nadir reflectance products from MODIS. Remote Sensing of Environment, 83, 135-148.

Tan, P., Lymburner, L., Mueller, M., Li, F., Thankappan, M. and Lewis, A. (2013). Applying machine learning methods and times series analysis to create a national dynamic land cover dataset for Australia. IEEE International Geoscience and Remote Sensing Symposium, Melbourne, Australia. 